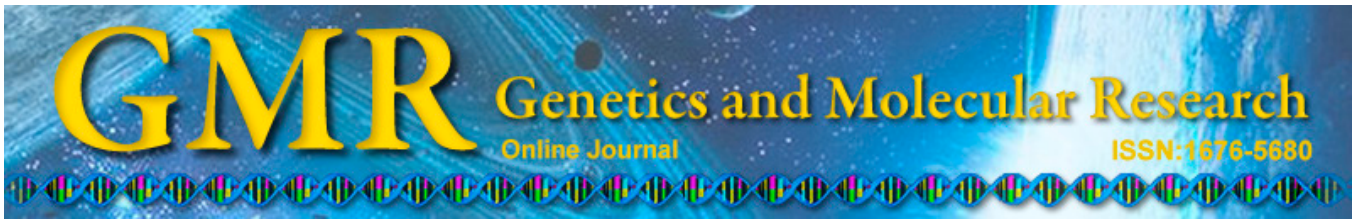

\title{
MicroRNA network analysis identifies key microRNAs and genes associated with precancerous lesions of gastric cancer
}

\author{
X.W. Wang ${ }^{1}$, Y. Wu ${ }^{2}$, D. Wang ${ }^{1}$ and Z.F. Qin ${ }^{1}$ \\ ${ }^{1}$ Department of Traditional Chinese Medicine, Shanghai Changzheng Hospital, \\ Second Military Medical University, Shanghai, China \\ ${ }^{2}$ Department of Gynecology of Traditional Chinese Medicine, \\ Shanghai Shuguang Hospital Baoshan Branch, Shanghai, China \\ Corresponding author: Z.F. Qin \\ E-mail: zhifengqin22@hotmail.com
}

Genet. Mol. Res. 13 (4): 8695-8703 (2014)

Received August 20, 2013

Accepted March 26, 2014

Published October 27, 2014

DOI http://dx.doi.org/10.4238/2014.October.27.10

\begin{abstract}
To identify potential targets for the early treatment and prevention of gastric cancer, microRNA (miRNA) expression profiles of precancerous lesions of gastric cancer were investigated. The miRNA microarray dataset GSE24839 was downloaded from Gene Expression Omnibus (GEO) and included 10 Helicobacter pylori-related gastritis samples and 10 gastric intestinal metaplasia samples. Differentially expressed miRNAs (DEMs) were screened using the Student $t$-test; $\mathrm{P}<0.05$ was considered to be statistically significant. Co-expression networks of total miRNAs and DEMs were constructed based on the Pearson correlation coefficient for the two diseases. Target genes of the DEMs were retrieved using miRecords and pathway-enrichment analysis was performed using a hypergeometric test. A total of 20 DEMs were obtained for $H$. pylori-related gastritis and gastric intestinal metaplasia samples, including 12 up-regulated and 8 down-regulated miRNAs. The identified DEMs appear to play key roles in gastric cancer, as the average degree of the DEM sub-network was higher than that of the
\end{abstract}


total miRNA co-expression network. Furthermore, target genes for 6 DEMs (hsa-miR-106b, hsa-miR-193a-3p, hsa-miR-204, hsa-miR-30e, hsa-miR-519d, and hsa-miR-524-5p) are in Kyoto Encyclopedia of Genes and Genomes (KEGG) pathways, including signal transduction, cell growth and death, and transport and catabolism. Among the target genes, 5 (RAB22A, SOX4, IKZF2, PLAG1, and BTBD7) were of interest because they can be simultaneously regulated by several DEMs. These findings suggest that these genes may be targets for modulating gastric cancer progression.

Key words: MicroRNAs; Gastric cancer; Differential expression; Network analysis

\section{INTRODUCTION}

Gastric cancer (GC) is a common gastrointestinal malignancy worldwide, resulting in an estimated 700,000 deaths annually (Fritz and Van der Merwe, 2009). GC develops through a cascade of well-defined stages (inflammation and atrophy, intestinal metaplasia, dysplasia, and carcinoma sequence) (Correa, 1988). The initial stages of gastritis and atrophy are attributed to infection by Helicobacter pylori (Hp) (Shiotani et al., 2010). Hp-related gastritis patients are at a high risk for developing $\mathrm{GC}$, which may gradually progress to gastric intestinal metaplasia (Sipponen and Hyvärinen, 1993; Correa, 1995; Ohata et al., 2004). Therefore, early diagnosis of patients with precancerous lesions is very important for preventing GC development.

Recently, specific microRNAs (miRNAs) have become potential biomarkers for cancer development (Leja et al., 2012). miRNAs can cause mRNA degradation or translational inhibition by full or partial complementary binding to 3'-untranslated regions (UTRs) of target mRNAs, thus regulating the expression of target genes (Lai, 2002; Jones-Rhoades and Bartel, 2004). These miRNAs take part in a series of important biological processes (Bartel and Bartel, 2003), such as proliferation, apoptosis, and cell differentiation, indicating that they may be useful for determining the underlying regulatory mechanisms in precancerous lesions of gastric cancer (PLGC) (Xiao et al., 2009a; Liu et al., 2010) and GC (Saito et al., 2009; Ueda et al., 2010). Several studies have also suggested that miRNAs represent the bridge between Hp-related gastritis and GC development (Matsushima et al., 2011; Zabaleta, 2012).

In the present study, we investigated the expression patterns of miRNAs in Hp-related gastritis and gastric intestinal metaplasia by analyzing miRNA microarray data using bioinformatic tools. Crucial miRNAs and their target genes were also screened, which may be potential targets for preventing and treating GC.

\section{MATERIAL AND METHODS}

\section{miRNA microarray data}

The miRNA microarray data set GSE24839 was downloaded from Gene Expression Omnibus (GEO). A total of 20 samples were extracted, including $10 \mathrm{Hp}$-related gastritis samples and 10 gastric intestinal metaplasia samples. Raw data were collected using GPL8871 OSU_CCC v4.0. 


\section{Preprocessing of raw data and differential analysis}

Raw data were preprocessed using the R software packages (Irizarry et al., 2003), including background subtraction, normalization, and summarization. Quantile normalization was conducted. If more than one probe corresponded to the same miRNA, their values were averaged and used as the final expression level. Differential analysis was carried out using the Student $t$-test; P $<0.05$ was set as the cut-off to screen for significantly differentially expressed miRNAs (DEMs).

\section{Construction of co-expression networks}

Co-expression networks were constructed for the miRNAs of Hp-related gastritis and gastric intestinal metaplasia. Pearson correlation coefficients (PCC) (Adler and Parmryd, 2010) were calculated for each interaction. A PCC with a $P$ value $<0.05$ was set as the threshold to filter out significant interactions.

\section{Retrieval of target genes}

Target genes of DEMs were predicted using miRecords (http://mirecords.umn.edu/ miRecords/index.php) (Xiao et al., 2009b). This site integrates 11 tools, including DIANAmicroT, MicroInspector, miRanda, MirTarget2, miTarget, NBmiRTar, PicTar, PITA, RNA22, RNAhybrid, and TargetScan/TargertScanS. miRNA-target gene interactions that were observed in no less than 4 tools were selected for further analysis.

\section{Pathway-enrichment analysis}

Pathway information was downloaded from the Kyoto Encyclopedia of Genes and Genomes (KEGG) (Kanehisa and Goto, 2000), which includes 232 pathways in 6 groups (cellular processes, environmental information processing, genetic information processing, human diseases, metabolism, and organismal systems). Pathway-enrichment analysis was carried out for the target genes using the following hypergeometric distribution-based statistical algorithm (Storey and Tibshirani, 2003):

$$
P=1-\sum_{i=0}^{k-1} \frac{\left(\begin{array}{l}
M \\
k
\end{array}\right)\left(\frac{N-M}{n-k}\right)}{\frac{N}{n}}
$$

where $N=$ number of genes in all pathways, $n=$ number of target genes, $M=$ number of genes in a specific pathway, and $k=$ number of overlapping genes.

\section{RESULTS}

\section{Co-expression networks}

Two co-expression networks were constructed based on PCCs, including one for Hp- 
related gastritis with 605 miRNAs (nodes) and 20,885 interactions (edges), and the other for gastric intestinal metaplasia with 605 nodes and 24,897 edges (Table 1).

Table 1. Co-expression networks for Helicobacter pylori (Hp)-related gastritis and gastric intestinal metaplasia.

\begin{tabular}{lccc}
\hline Parameters & Hp-related gastritis network & Gastric intestinal metaplasia network & P value (K-S test) \\
\hline Nodes & 605 & 605 & 24,897 \\
Edges & 20,885 & 78.16954 & $9.691 \mathrm{e}-11$ \\
Average degree & 65.573 & 396.2936 & 0.1835 \\
Average betweenness & 408.7473 & $6.936105 \mathrm{e}-06$ & 0.6635 \\
Average closeness & $6.992293 \mathrm{e}-06$ & & \\
\hline
\end{tabular}

The miRNAs identified in the two networks were identical, suggesting that the same miRNAs are involved in progression of the two diseases. However, a larger number of edges was observed in gastric intestinal metaplasia compared with Hp-related gastritis. The average degrees between miRNAs for gastric intestinal metaplasia and Hp-related gastritis were 78.16954 and 65.573, respectively, which was a significant difference $(\mathrm{P}=9.691 \mathrm{e}-11)$. The betweenness and closeness for the network of Hp-related gastritis were slightly larger than those of gastric intestinal metaplasia, but no significant difference was observed. Betweenness quantifies the number of times a node acts as a bridge along the shortest path between 2 other nodes. Closeness reflects the degree of interaction between the current node and other nodes.

\section{DEMs and co-expression sub-networks}

DEMs between Hp-related gastritis and gastric intestinal metaplasia were screened using the Student $t$-test. To control the false-positive rate but to identify a larger number of DEMs, a P value $<0.05$ was selected as the cut-off point. A total of 20 DEMs were acquired, including 12 up-regulated and 8 down-regulated miRNAs.

DEM-related co-expression networks were extracted from whole networks. The subnetwork for Hp-related gastritis was comprised of 494 nodes and 1287 edges (Figure 1), while that for gastric intestinal metaplasia contained 498 nodes and 1085 edges (Figure 2). The average degree between miRNAs of gastric intestinal metaplasia was significantly lower than that of Hp-related gastritis (4.3574 vs 5.2105, $\mathrm{P}=2.0674 \mathrm{e}-004)$. Similarly, the average degree of DEMs for gastric intestinal metaplasia was also smaller than that for Hp-related gastritis (55.1000 vs 65.1000, $\mathrm{P}=3.256 \mathrm{E}-03)$.

Further analysis showed that a high degree was present in some of the DEMs, suggesting that these DEMs may act as key players in networks. The degrees for each DEM in the sub-network of gastric intestinal metaplasia are listed in Table 2. The top 5 DEMs were hsa-miR-486-5p, hsa-miR-645, hsa-miR-624*, hsa-miR-504, and hsa-miR-106b.

\section{Pathway-enrichment analysis results}

Target genes were retrieved using miRecords, after which pathway-enrichment analysis was performed using a hypergeometric distribution-based test. A P value $<0.05$ was chosen as the significance threshold to ensure the reliability of the results. Next, the target genes of 6 DEMs (hsa-miR-106b, hsa-miR-193a-3p, hsa-miR-204, hsa-miR-30e, hsa-miR-519d, and hsa-miR-524-5p) were enriched to KEGG pathways (Table 3), including signal transduction 
pathways (e.g., "transforming growth factor $\beta$ (TGF- $\beta$ ) signaling pathway", "MAPK signaling pathway", "calcium signaling pathway", and "neurotrophin signaling pathway"), cell growth and death pathways (e.g., "cell cycle"), as well as transport and catabolism pathways (e.g., "endocytosis"). These pathways have been linked to cancers, and any abnormalities imply a high risk for GC.

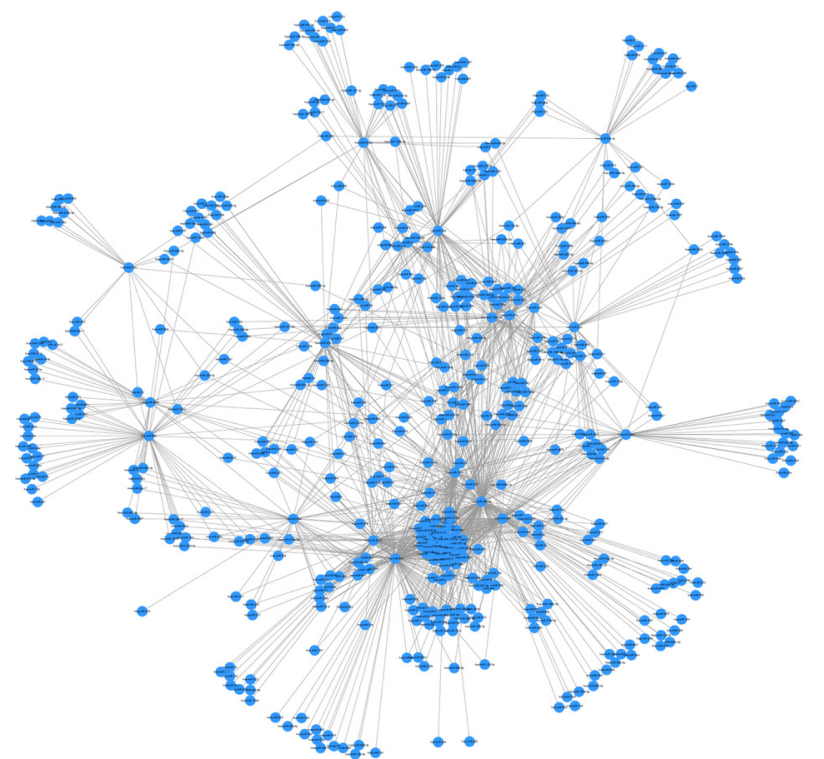

Figure 1. Co-expression network for miRNAs in Helicobacter pylori-related gastritis.

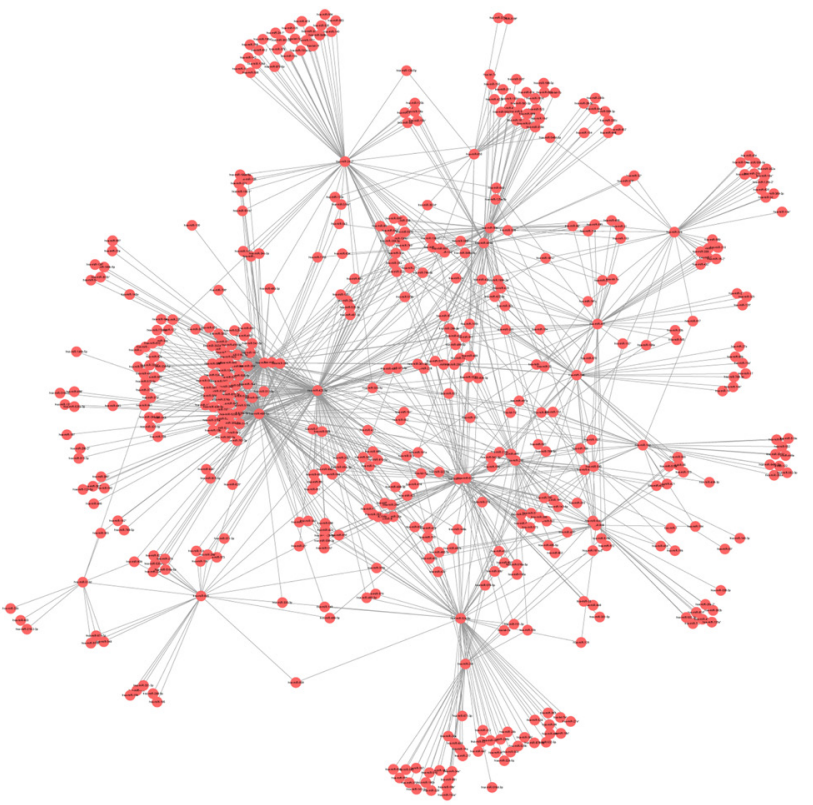

Figure 2. Co-expression network for miRNAs in gastric intestinal metaplasia. 
Table 2. Degrees of differentially expressed miRNAs in the sub-network of gastric intestinal metaplasia.

\begin{tabular}{lc}
\hline miRNA & Degree \\
\hline hsa-miR-486-5p & 196 \\
hsa-miR-645 & 146 \\
hsa-miR-624* & 128 \\
hsa-miR-504 & 97 \\
hsa-miR-106b & 94 \\
hsa-miR-24-2* & 84 \\
hsa-miR-198 & 67 \\
hsa-miR-193a-3p & 65 \\
hsa-miR-215 & 59 \\
hsa-miR-650 & 49 \\
hsa-miR-423-5p & 49 \\
hsa-miR-204 & 38 \\
hsa-miR-608 & 37 \\
hsa-miR-595 & 36 \\
hsa-miR-519d & 33 \\
hsa-miR-192 & 29 \\
hsa-miR-451 & 27 \\
hsa-miR-450a & 27 \\
hsa-miR-524-5p & 24 \\
hsa-miR-30e & 17 \\
\hline
\end{tabular}

Table 3. Significantly over-represented pathways for the 6 differentially expressed miRNAs.

\begin{tabular}{lccl}
\hline microRNA & Target & Pathway & Key pathways \\
\hline hsa-miR-106b & 1182 & 25 & Pathways in cancer, TGF- $\beta$ signaling pathway, MAPK signaling pathway \\
hsa-miR-193a-3p & 236 & 7 & Neurotrophin signaling pathway, MAPK signaling pathway, pathways in cancer \\
hsa-miR-204 & 761 & 6 & Calcium signaling pathway, neurotrophin signaling pathway, axon guidance \\
hsa-miR-30e & 1304 & 18 & Axon guidance, ubiquitin-mediated proteolysis, neurotrophin signaling pathway \\
hsa-miR-519d & 856 & 7 & Axon guidance, MAPK signaling pathway, pathways in cancer \\
hsa-miR-524-5p & 617 & 6 & Endocytosis, cell cycle, TGF- $\beta$ signaling pathway \\
\hline
\end{tabular}

Target $=$ number of target genes; Pathway = number of significantly over-represented pathways; Key pathways = some examples of the significantly over-represented pathways.

In addition, 5 genes were of particular interest because they could be simultaneously regulated by several DEMs; these genes included RAB22A (member of the RAS oncogene family), SRY (sex-determining region Y)-box 4 (SOX4), IKAROS family zinc finger 2 (Helios) (IKZF2), pleiomorphic adenoma gene 1 (PLAG1), and BTB (POZ) domain containing 7 (BTBD7). These genes may be biomarkers of cancers and thus should be further examined.

\section{DISCUSSION}

In the present study, miRNA microarray data for Hp-related gastritis and gastric intestinal metaplasia were analyzed to investigate miRNA expression patterns and provide insight into their pathogenesis. Co-expression networks were established for the 2 diseases, respectively. The average degree in the network for gastric intestinal metaplasia was significantly higher than that for Hp-related gastritis, suggesting that interactions between miRNAs became more frequent as the disease developed. However, for sub-networks based upon DEMs, a significantly higher average degree was observed in Hp-related gastritis, implying that these DEMs exert their roles during early disease stages. These co-expression networks and analysis results provide valuable information regarding the mechanisms regulating disease progression. 
To identify key players in the network, 20 DEMs important in the 2 diseases were screened. A high degree was observed in some of the DEMs, confirming their hub roles in regulatory networks. Target genes were predicted for DEMs and then pathway-enrichment analysis was carried out to examine the biological functions of the DEMs. Only the target genes of 6 DEMs (hsa-miR-106b, hsa-miR-193a-3p, hsa-miR-204, hsa-miR-30e, hsa-miR-519d, and hsa-miR-524-5p) were enriched in pathways associated with GC development. Matsushima et al. (2011) observed lower expression of miR-106b and miR-204 in the Hp-positive group compared to the Hp-negative group, suggesting that the down-regulation of these miRNAs is associated with Hp-related chronic gastritis. In addition, miR-106b has been reported to suppress TGF- $\beta$-induced cell cycle arrest and promote GC development (Petrocca et al., 2008). miR-204 could down-regulate sirtuin 1 (SIRT1) and thus revert the SIRT1-induced epithelial-mesenchymal transition and invasion in GC cells (Zhang et al., 2013). Down-regulation of miR-204 is linked to enhanced cell proliferation (Lee et al., 2010). miR-193a has been shown to inhibit cellular transformation as well as tumor growth by directly targeting the 3'-UTR of plasminogen activator, urokinase (PLAU) and v-Ki-ras2 Kirsten rat sarcoma viral oncogene homolog (K-Ras) (Iliopoulos et al., 2011). Furthermore, Kozaki et al. (2008) found that miR-193a down-regulation via DNA hypermethylation may accelerate carcinogenesis. miR-30 expression can be increased by the beta-catenin/TCF4 complex during intestinal cell differentiation (Liao and Lönnerdal, 2010), and over-expression of miR-519d promotes cell proliferation and invasion as well as impairs apoptosis by directly targeting cyclin-dependent kinase inhibitor 1A (CDKN1A/p21) in cancer (Fornari et al., 2012). miR-524-5p has been demonstrated to suppress cancer cell proliferation and invasion by down-regulating Jagged-1 and Hes-1, two key components of the Notch signaling pathway (Chen et al., 2012). In this study, we found that miR-524-5p may be involved in GC by regulating the cell cycle and the TGF- $\beta$ signaling pathway.

Moreover, 5 genes simultaneously targeted by several miRNAs were screened for their potential as therapeutic targets to treat gastritis and GC. RAB22A is a member of the RAB family of small GTPases that are predominantly associated with endosome trafficking. Endosomes play key roles in the control of cell signaling, the epithelial to mesenchymal transition, and cell adhesion and migration, thus influencing cancer development (Lanzetti and Di Fiore, 2008). Several studies have demonstrated that members of the RAB family are up-regulated in GC tissues (Hou et al., 2008; Cao et al., 2013). SOX4 is a member of the SRYrelated HMG-box (SOX) family of transcription factors involved in cell apoptosis as well as tumorigenesis. SOX4 is up-regulated in GC compared to benign gastric tissues, which may be attributed to the hypermethylation of miR-129 (Shen et al., 2010). PLAG1 is a zinc-finger protein with 2 putative nuclear localization signals. Studies in salivary gland tumors have shown that PLAG1 can induce the expression of a number of growth factors that eventually promote tumor growth and development (Stenman, 2005; Asp et al., 2006). BTBD7 regulates epithelial-mesenchymal transition-associated proteins and promotes hepatocellular carcinogenesis, angiogenesis, and metastasis (Tao et al., 2013). The close relationships between these genes and cancers demonstrate the validity of our method.

Overall, miRNA expression patterns for the two types of PLGCs were investigated using miRNA microarray data. Using bioinformatic tools, a number of key miRNAs and genes closely related to GC were identified, implying their potential applications in the prevention and treatment of GC. 


\title{
Conflicts of interest
}

\author{
The authors declare no conflict of interest.
}

\section{REFERENCES}

Adler J and Parmryd I (2010). Quantifying colocalization by correlation: the Pearson correlation coefficient is superior to the Mander's overlap coefficient. Cytometry A 77: 733-742.

Asp J, Persson F, Kost-Alimova M and Stenman G (2006). CHCHD7-PLAG1 and TCEA1-PLAG1 gene fusions resulting from cryptic, intrachromosomal 8q rearrangements in pleomorphic salivary gland adenomas. Genes Chromosomes Cancer 45: 820-828.

Bartel B and Bartel DP (2003). MicroRNAs: at the root of plant development? Plant Physiol. 132: 709-717.

Cao C, Lu C, Xu J, Zhang J, et al. (2013). Expression of Rab25 correlates with the invasion and metastasis of gastric cancer. Chin. J. Cancer Res. 25: 192-199.

Chen L, Zhang W, Yan W, Han L, et al. (2012). The putative tumor suppressor miR-524-5p directly targets Jagged-1 and Hes-1 in glioma. Carcinogenesis 33: 2276-2282.

Correa P (1988). A human model of gastric carcinogenesis. Cancer Res. 48: 3554-3560.

Correa P (1995). Helicobacter pylori and gastric carcinogenesis. Am. J. Surg. Pathol. 19 (Suppl 1): S37-S43.

Fornari F, Milazzo M, Chieco P, Negrini M, et al. (2012). In hepatocellular carcinoma miR-519d is up-regulated by p53 and DNA hypomethylation and targets CDKN1A/p21, PTEN, AKT3 and TIMP2. J. Pathol. 227: 275-285.

Fritz EL and Van der Merwe SW (2009). Helicobacter pylori and gastric cancer. CME 27: 220-222.

Hou Q, Wu YH, Grabsch H, Zhu Y, et al. (2008). Integrative genomics identifies RAB23 as an invasion mediator gene in diffuse-type gastric cancer. Cancer Res. 68: 4623-4630.

Iliopoulos D, Rotem A and Struhl K (2011). Inhibition of miR-193a expression by Max and RXRalpha activates K-Ras and PLAU to mediate distinct aspects of cellular transformation. Cancer Res. 71: 5144-5153.

Irizarry RA, Hobbs B, Collin F, Beazer-Barclay YD, et al. (2003). Exploration, normalization, and summaries of high density oligonucleotide array probe level data. Biostatistics 4: 249-264.

Jones-Rhoades MW and Bartel DP (2004). Computational identification of plant microRNAs and their targets, including a stress-induced miRNA. Mol. Cell 14: 787-799.

Kanehisa M and Goto S (2000). KEGG: Kyoto Encyclopedia of Genes and Genomes. Nucleic Acids Res. 28: 27-30.

Kozaki K, Imoto I, Mogi S, Omura K, et al. (2008). Exploration of tumor-suppressive microRNAs silenced by DNA hypermethylation in oral cancer. Cancer Res. 68: 2094-2105.

Lai EC (2002). Micro RNAs are complementary to 3' UTR sequence motifs that mediate negative post-transcriptional regulation. Nat. Genet. 30: 363-364.

Lanzetti L and Di Fiore PP (2008). Endocytosis and cancer: an 'insider' network with dangerous liaisons. Traffic 9: 20112021.

Lee Y, Yang X, Huang Y, Fan H, et al. (2010). Network modeling identifies molecular functions targeted by miR-204 to suppress head and neck tumor metastasis. PLoS Comput. Biol. 6: e1000730.

Leja M, Wex T and Malfertheiner P (2012). Markers for gastric cancer premalignant lesions: where do we go? Dig. Dis. 30: $268-276$.

Liao Y and Lönnerdal B (2010). Beta-catenin/TCF4 transactivates miR-30e during intestinal cell differentiation. Cell Mol. Life Sci. 67: 2969-2978.

Liu Z, Xiao B, Tang B, Li B, et al. (2010). Up-regulated microRNA-146a negatively modulate Helicobacter pyloriinduced inflammatory response in human gastric epithelial cells. Microbes Infect. 12: 854-863.

Matsushima K, Isomoto H, Inoue N, Nakayama T, et al. (2011). MicroRNA signatures in Helicobacter pylori-infected gastric mucosa. Int. J. Cancer 128: 361-370.

Ohata H, Kitauchi S, Yoshimura N, Mugitani K, et al. (2004). Progression of chronic atrophic gastritis associated with Helicobacter pylori infection increases risk of gastric cancer. Int. J. Cancer 109: 138-143.

Petrocca F, Vecchione A and Croce CM (2008). Emerging role of miR-106b-25/miR-17-92 clusters in the control of transforming growth factor beta signaling. Cancer Res. 68: 8191-8194.

Saito Y, Suzuki H and Hibi T (2009). The role of microRNAs in gastrointestinal cancers. J. Gastroenterol. 44 (Suppl 19): 18-22.

Shen R, Pan S, Qi S, Lin X, et al. (2010). Epigenetic repression of microRNA-129-2 leads to overexpression of SOX4 in gastric cancer. Biochem. Biophys. Res. Commun. 394: 1047-1052. 
Shiotani A, Nishi R, Uedo N, Iishi H, et al. (2010). Helicobacter pylori eradication prevents extension of intestinalization even in the high-risk group for gastric cancer. Digestion 81: 223-230.

Sipponen P and Hyvärinen H (1993). Role of Helicobacter pylori in the pathogenesis of gastritis, peptic ulcer and gastric cancer. Scand. J. Gastroenterol. Suppl. 196: 3-6.

Stenman G (2005). Fusion oncogenes and tumor type specificity-insights from salivary gland tumors. Semin. Cancer Biol. 15: 224-235.

Storey JD and Tibshirani R (2003). Statistical significance for genomewide studies. Proc. Natl. Acad. Sci. U. S. A. 100: 9440-9445.

Tao YM, Huang JL, Zeng S, Zhang S, et al. (2013). BTB/POZ domain-containing protein 7: epithelial-mesenchymal transition promoter and prognostic biomarker of hepatocellular carcinoma. Hepatology 57: 2326-2337.

Ueda T, Volinia S, Okumura H, Shimizu M, et al. (2010). Relation between microRNA expression and progression and prognosis of gastric cancer: a microRNA expression analysis. Lancet Oncol. 11: 136-146.

Xiao B, Liu Z, Li BS, Tang B, et al. (2009a). Induction of microRNA-155 during Helicobacter pylori infection and its negative regulatory role in the inflammatory response. J. Infect. Dis. 200: 916-925.

Xiao F, Zuo Z, Cai G, Kang S, et al. (2009b). miRecords: an integrated resource for microRNA-target interactions. Nucleic Acids Res. 37: D105-D110.

Zabaleta J (2012). MicroRNA: A bridge from H. pylori infection to gastritis and gastric cancer development. Front Genet. 3: 294.

Zhang L, Wang X and Chen P (2013). MiR-204 down regulates SIRT1 and reverts SIRT1-induced epithelial-mesenchymal transition, anoikis resistance and invasion in gastric cancer cells. BMC Cancer 13: 290. 